\title{
Measuring Aspect Cohesion
}

\author{
Jianjun Zhao ${ }^{1}$ and Baowen $\mathrm{Xu}^{2}$
}

1 Department of Computer Science and Engineering, Fukuoka Institute of Technology 3-30-1 Wajiro-Higashi, Fukuoka 811-0295, Japan

zhao@cs.fit.ac.jp

2 Department of Computer Science and Engineering, Southeast University

Nanjing 210096, China

bwxu@cs.seu.edu.cn

\begin{abstract}
Cohesion is an internal software attribute representing the degree to which the components are bound together within a software module. Cohesion is considered to be a desirable goal in software development, leading to better values for external attributes such as maintainability, reusability, and reliability. Aspect-oriented software development (AOSD) is a new technique to support separation of concerns in software development. AOSD introduces a new kind of component called aspect which is like a class, also consisting of attributes (aspect instance variables) and those modules such as advice, introduction, pointcuts, and methods. The cohesion for such an aspect is therefore mainly about how tightly the attributes and modules of aspects cohere. To test this hypothesis, cohesion measures for aspects are needed. In this paper, we propose an approach to assessing the aspect cohesion based on dependence analysis. To this end, we present various types of dependencies between attributes and/or modules in an aspect, and the aspect dependence graph (ADG) to explicitly represent these dependencies. Based on the ADG, we formally define some aspect cohesion measures. We also discuss the properties of these dependencies, and according to these properties, we prove that these measures satisfy the properties that a good measure should have.
\end{abstract}

\section{Introduction}

Aspect-oriented software development (AOSD) is a new technique to support separation of concerns in software development [2/12 1314]. The techniques of AOSD make it possible to modularize crosscutting aspects of a system. Aspects in AOSD may arise at any stage of the software life cycle, including requirements specification, design, implementation, etc. Some examples of crosscutting aspects are exception handling, synchronization, and resource sharing.

The current research so far in AOSD is focused on problem analysis, software design, and implementation techniques. However, efficient evaluations of this new design technique in a rigorous and quantitative fashion are still ignored during the current stage of the technical development. For example, it has been frequently claimed that applying an AOSD method will eventually lead to quality 
software, but unfortunately, there is little data to support such claim. Aspectoriented software is supposed to be easy to maintain, reuse, and evolve, yet few quantitative studies have been conducted, and measures to quantify the amount of maintenance, reuse, and evolution in aspect-oriented systems are lacking. In order to verify claims concerning the maintainability, reusability, and reliability of systems developed using aspect-oriented techniques, software measures are required.

As with procedural and object-oriented software, we should also relate aspectoriented structural quality to some critical process attributes concerning software maintainability, reusability, and reliability. We therefore need appropriate measures of aspect-oriented structure to begin to relate structure to process. Recently, Zhao developed a suite of dependence-based structural measures which are specifically designed to quantify the information flows in aspect-oriented software 16].

Cohesion is a structural attribute whose importance is well-recognized in the software engineering community 4815. Cohesion is an internal software attribute representing the degree to which the components are bound together within a software module. Cohesion is considered to be a desirable goal in software development, which may lead to better values for external attributes such as maintainability, reusability, and reliability. In procedural or object-oriented paradigm, a highly cohesive component is one with one basic function and is difficult to be decomposed. Cohesion is therefore considered to be a desirable goal in software development, leading to better values for external attributes such as maintainability, reusability, and reliability. A system should have high cohesion. Recently, many cohesion measures and several guidelines to measure cohesion of a component have been developed for procedural software 311 and for object-oriented software $[6,8,10,4,5]$.

Aspect-oriented language introduces a new kind of component called aspect to model the crosscutting concerns in a software system. An aspect with its encapsulation of state (attributes) and associated modules (operations) such as advice, introduction, pointcuts, and methods is a different abstraction in comparison to a procedure within procedural systems and a class within objectoriented systems. The cohesion of an aspect is therefore mainly about how tightly the aspect's attributes and modules cohere.

However, although cohesion has been studied widely for procedural and object-oriented software, it has not been studied for aspect-oriented software yet. Since an aspect contains new modules such as advice, introduction, and pointcuts that are different from methods in a class, existing class cohesion measures can not be directly applied to aspects. Therefore, new measures that are appropriate for measuring aspect cohesion are needed.

In this paper, we propose an approach to assessing the aspect cohesion based on dependence analysis. To this end, we present various types of dependencies between attributes and/or modules such as advice, introduction, pointcuts, and methods of an aspect, and a dependence-based representation called aspect dependence graph (ADG) to represent these dependencies. Based on the ADG, we formally define some aspect cohesion measures. We also discuss the properties 
of these dependencies, and according to these properties, we prove that these measures satisfy the properties that a good measure should have.

We hope that by studying the ideas of aspect cohesion from several different viewpoints and through well developed cohesion measures, we can obtain a better understanding of what the cohesion is meant in aspect-oriented paradigm and the role that cohesion plays in the development of quality aspect-oriented software. As the first step to study the aspect cohesion, the goal of this paper is to provide a sound and formal basis for aspect cohesion measures before applying them to real aspect-oriented software design.

The rest of the paper is organized as follows. Section 2 briefly introduces AspectJ, a general aspect-oriented programming language based on Java. Section 3 defines three types of dependencies in an aspect and discusses some basic properties of these dependencies. Section 4 proposes an approach to measuring aspect cohesion from three facets: inter-attribute, module-attribute and intermodule. Section 5 discusses some related work. Concluding remarks are given in Section 6

\section{Aspect-Oriented Programming and AspectJ}

We present our basic ideas of aspect cohesion measurement approach for aspectoriented programs in the context of AspectJ, the most widely used aspectoriented programming language [1]. Our basic ideas, however, deal with the basic concepts of aspect-oriented programming and therefore apply to the general class of aspect-oriented languages.

AspectJ [1] is a seamless aspect-oriented extension to Java. AspectJ adds to Java some new concepts and associated constructs. These concepts and associated constructs are called join point, pointcut, advice, introduction, and aspect.

Aspect is a modular unit of crosscutting implementation in AspectJ. Each aspect encapsulates functionality that crosscuts other classes in a program. An aspect is defined by aspect declaration, which has a similar form of class declaration in Java. Similar to a class, an aspect can be instantiated and can contain state and methods, and also may be specialized in its sub-aspects. An aspect is then combined with the classes it crosscuts according to specifications given within the aspect. Moreover, an aspect can introduce methods, attributes, and interface implementation declarations into types by using the introduction construct. Introduced members may be made visible to all classes and aspects (public introduction) or only within the aspect (private introduction), allowing one to avoid name conflicts with pre-existing members.

The essential mechanism provided for composing an aspect with other classes is called a join point. A join point is a well-defined point in the execution of a program, such as a call to a method, an access to an attribute, an object initialization, exception handler, etc. Sets of join points may be represented by pointcuts, implying that such sets may crosscut the system. Pointcuts can be composed and new pointcut designators can be defined according to these combinations. AspectJ provides various pointcut designators that may be combined through logical operators to build up complete descriptions of pointcuts of interest. For a complete listing of possible designators one can refer to [1]. 


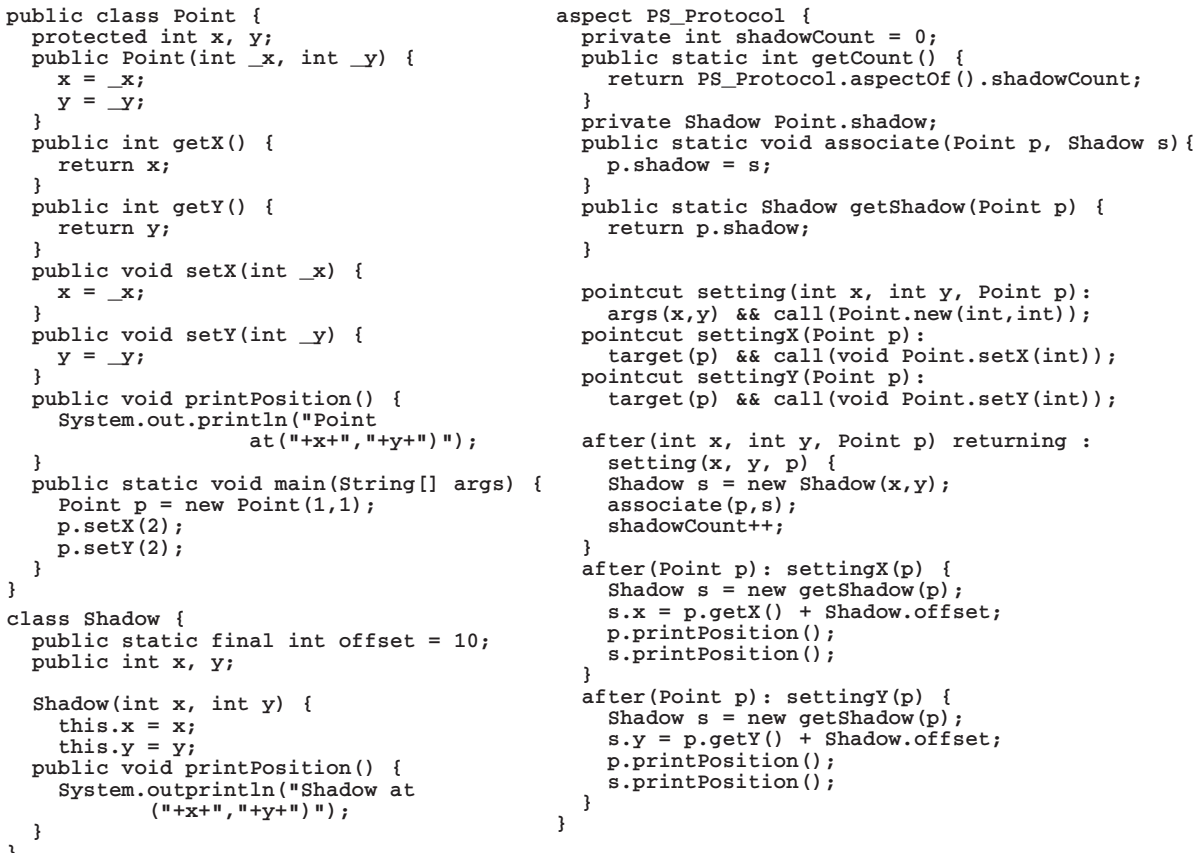

Fig. 1. A sample AspectJ program.

An aspect can specify advice that is used to define some code that should be executed when a pointcut is reached. Advice is a method-like mechanism which consists of code that is executed before, after, or around a pointcut. Around advice executes in place of the indicated pointcut, allowing a method to be replaced.

An AspectJ program can be divided into two parts: base code part which includes classes, interfaces, and other language constructs for implementing the basic functionality of the program, and aspect code part which includes aspects for modeling crosscutting concerns in the program. Moreover, any implementation of AspectJ should ensure that the base and aspect code run together in a properly coordinated fashion. Such a process is called aspect weaving and involves making sure that applicable advice runs at the appropriate join points. For detailed information about AspectJ, one can refer to [1].

Example. Fig. 1 shows an AspectJ program that associates shadow points with every Point object. The program contains one aspect PS_Protocol and two classes Point and Shadow. The aspect has three methods getCount, associate and getShadow and three pieces of advice related to pointcuts setting, settingX and settingY respectively. The aspect also has two attributes, i.e., shadowCount which is an attribute of the aspect itself and shadow which is an attribute that is privately introduced to class Point.

${ }^{1}$ Since advice in AspectJ has no name. So for easy expression, we use the name of a pointcut to stand for the name of advice it associated with. 
In the rest of the paper, we use this example to demonstrate our basic ideas of aspect cohesion measurement. We also assume that an aspect is composed of attributes (aspect instance variables), and modules 2 such as advice, introduction, pointcuts and methods.

\section{Aspect Dependencies}

In this section we define various types of dependencies between modules and/or attributes in an aspect and discuss some properties of these dependencies.

\subsection{Dependence Definitions}

We define three types of dependencies between attributes and/or modules in an aspect, that is, inter-attribute, inter-module, and module-attribute dependence.

Definition 1. Let $a_{1}, a_{2}$ be attributes in an aspect. $a_{2}$ is inter-attribute dependent on $a_{1}$, denoted by $a_{2} \hookrightarrow a_{1}$, if one of the following conditions holds:

- The definition of $a_{2}$ uses (refers) $a_{1}$ directly or indirectly, or

- Whether $a_{2}$ can be defined is determined by the state of $a_{1}$.

Generally, if $a_{2}$ is used in the condition part of a control statement (such as if and while), and the definition of $a_{1}$ is in the inner statement of the control statement, then the definition of $a_{1}$ depends on the state of $a_{2}$. For example, according to Definition 1, we know that there is no inter-attribute dependence in aspect PS_Protocol of Fig. 1.

There are two types of dependencies between aspect modules: inter-module call dependence and inter-module potential dependence.

Definition 2. Let $m_{1}, m_{2}$ be two modules and a be an attribute in an aspect. $m_{2}$ is inter-module-dependent on $m_{1}$, denoted by $m_{2} \rightarrow m_{1}$, if one of the following conditions holds:

- $m_{1}$ is called in $m_{2}$. (inter-module call dependence)

- $a$ is used in $m_{2}$ before it is defined, and a is defined in $m_{1}$. (inter-module potential dependence)

Given an aspect, we can not assume which piece of introduction or which method in the aspect might be invoked before another 3 . So we assume that all the introduction and methods in the aspect can be invoked at any time and in any order. Therefore, if $m_{2}$ might use an attribute $a$, and $a$ is defined in $m_{1}$, and if $m_{1}$ is invoked first and then $m_{2}$ is invoked, then $m_{2}$ might use $a$ defined in $m_{1}$, i.e. $m_{2}$ is inter-module potentially-dependent on $m_{1}$.

\footnotetext{
${ }^{2}$ For unification, we use the word "module" to stand for a piece of advice, a piece of introduction, a pointcut, or a method declared in an aspect.

${ }^{3}$ In AspectJ, advice is automatically woven into some methods in a class by the compiler, and therefore no call exists for the advice.
} 
To obtain the inter-module dependencies, for each module $m$, we introduce two sets, $D_{I N}$ and $D_{O U T}$, where $D_{I N}(m)$ is the set of attributes referred before modifying their values in $m$, and $D_{O U T}(m)$ is the set of attributes modified in $m$. Thus, for an attribute $a$ and modules $m$ and $m^{\prime}$, if $a \in D_{I N}\left(m^{\prime}\right) \cap D_{O U T}(m)$, then $m^{\prime} \rightarrow m$.

In addition to attributes, since there are four types of different modules in an aspect, i.e., advice, introduction, pointcuts and methods, there may have the following possible types of inter-module dependencies, i.e., dependencies between advice and advice, advice and introduction, advice and method, advice and pointcut 4 , introduction and introduction, introduction and method, or method and method.

Example. In order to compute inter-module dependencies in aspect PS_Protocol, we first compute the $D_{I N}$ and $D_{O U T}$ sets for each module in PS_Protocol. They are: $D_{I N}$ (getCount $)=\{$ shadowCount $\}, D_{O U T}$ (getCount $)=\emptyset, D_{I N}$ (getShadow $)=$ $\{$ shadow $\}, D_{\text {OUT }}($ getShadow $)=\emptyset, D_{I N}($ associate $)=\emptyset, D_{O U T}($ associate $)=\{$ sha dow $\}, D_{I N}($ setting $)=\{$ shadowCount $\}, D_{O U T}$ (setting $)=\{$ shadowCount $\}, D_{I N}($ set tingX $)=D_{O U T}($ setting $)=\emptyset, D_{I N}($ setting $Y)=D_{O U T}($ setting $\mathrm{Y})=\emptyset$. Also there exist inter-module dependencies between each pointcut and its corresponding advice. So we finally get the following inter-module dependencies in PS_Protocol.

(method getCount $\rightarrow$ advicesetting), (advice setting $\rightarrow$ method associate), (advice settingX $\rightarrow$ method getShadow), (advice settingY $\rightarrow$ advice getShadow), (pointcut setting $\rightarrow$ advice setting), (pointcut settingX $\rightarrow$ advice settingX), and (pointcut setting $\rightarrow$ advice settingX).

Definition 3. Let $m$ be a module and a be an attribute in an aspect. $m$ is module-attribute-dependent on a, denoted by $m \mapsto a$, if a is referred in $m$.

Since there are four types of different modules in an aspect, module-attribute dependencies may have four different types: advice-attribute, introduction-attribute, pointcut-attribute, or method-attribute dependencies.

Example. According to Definition [3, the module-attribute dependencies in aspect PS_Protocol are: (method getCount $\mapsto$ attribute shadowCount), (method getShadow $\mapsto$ attribute shadowCount), (advice settingX $\mapsto$ attribute shadowCount).

Note that all these types of dependencies defined above can be derived by performing control flow and data flow analysis using existing flow analysis algorithms 17. Due to the space limitation, we do not discuss this issue here.

\subsection{Dependence Properties}

This section discusses some properties of dependencies defined in Section 3.1 and refines the definition of inter-module dependence to fit for measuring aspect cohesion.

\footnotetext{
${ }^{4}$ A pointcut is only related to its corresponding advice. Therefore, there is no dependence between pointcut and method, pointcut and introduction, or pointcut and pointcut.
} 
According to definition [1, if $a_{1} \hookrightarrow a_{2}$ and $a_{2} \hookrightarrow a_{3}$, then $a_{1} \hookrightarrow a_{3}$. Therefore, we have

Property 1. The inter-attribute dependencies are transitive.

Based on Property [1, we can define the inter-attribute transitive dependence as follows.

Definition 4. Let $A$ be an aspect and $a_{i}(i>0)$ be attributes in $A$. If there exist attributes $a_{1}, a_{2}, \ldots, a_{n-1}, a_{n}(n>1)$, where $a_{1} \hookrightarrow a_{2}, \ldots, a_{i-1} \hookrightarrow a_{i}, \ldots$, $a_{n-1} \hookrightarrow a_{n}$, then $a_{1}$ is inter-attribute-transitive-dependent on $a_{n}$, denoted by $a_{1} \stackrel{*}{\hookrightarrow} a_{n}$.

According to definition 2, for modules $m_{1}, m_{2}$, and $m_{3}$, if $m_{1} \rightarrow m_{2}$ and $m_{2} \rightarrow m_{3}$, then $m_{1} \rightarrow m_{3}$ may not hold. Consider an example of inter-module call dependence, if $m_{1}$ is called in $m_{2}$ and $m_{2}$ is called in $m_{3}$, then $m_{1}$ is not necessarily called in $m_{3}$. For inter-module potential dependence, if $m_{1} \rightarrow m_{2}$ and $m_{2} \rightarrow m_{3}$ are introduced by unrelated, different attributes, then $m_{1}$ might have no relation with $m_{3}$. Therefore, we have

Property 2. The inter-module dependencies are not transitive.

The intransitivity among inter-module dependencies leads to great difficulties when performing analysis. Thus, we should redefine the inter-module dependencies.

Definition 5. Let $m_{1}, m_{2}$ be modules and a be an attribute in an aspect. If a is used in $m_{1}$ and defined in $m_{2}$, then a used in $m_{1}$ is dependent on a defined in $m_{2}$, denoted by $m_{1} \stackrel{a, a}{\longrightarrow} m_{2}$, where $<a$, a $>$ is named as a tag.

For unification, add a tag $\left.<^{*}, *^{*}\right\rangle$ for each inter-module call dependence arc, i.e., if $m_{1}$ is inter-module-call-dependent on $m_{2}$, then we have $m_{1} \stackrel{*, *}{\longrightarrow} m_{2}$.

Definition 5 is the basic definition. Since the dependencies between attributes are transitive, we can obtain a more general definition according to Property 3.

To obtain such dependencies, we introduce two sets for each module $m$ of an aspect, i.e., $D_{A}$ and $D_{A O}$, each element of which has the form $\left(a, a^{\prime}\right)$, where $a$ and $a^{\prime}$ are attributes of the aspect.

- $D_{A}(m)$ is the set of dependencies which records the dependencies from the attributes referred in $m$ to the attributes defined out $m . D_{A}(m)$ is a subset of inter-attribute dependencies.

- $D_{A O}(m)$ is the set of dependencies which records the dependencies from the attributes referred in $m$ to the attribute defined out $m$ when exiting $m$.

In general, the intermediate results are invisible outside, and an attribute might be modified many times in a piece of advice, a piece of introduction, or a method. We introduce $D_{A O}$ to improve the precision. Obviously, we have $D_{A O}(m) \subseteq D_{A}(m)$. 
Definition 6. Let $m_{1}, m_{2}$ be modules and $a_{1}, a_{2}$ be attributes in an aspect. If $\left(a_{1}, a_{2}\right) \in D_{A}\left(m_{1}\right)$ and $a_{2} \in D_{\text {OUT }}\left(m_{2}\right)$, then $m_{1}$ is dependent on $m_{2}$, denoted by $m_{1} \stackrel{a_{1}, a_{2}}{\longrightarrow} m_{2}$.

According to Definition [6] we have the following properties:

Property 3. Let $m_{1}, m_{2}, m_{3}$ be modules and $a_{1}, a_{2}, a_{3}$ be attributes in an aspect. If $m_{1} \stackrel{a_{1}, a_{2}}{\longrightarrow} m_{2}$, and $\forall\left(a_{2}, a_{3}\right),\left(a_{2}, a_{3}\right) \in D_{A O}\left(m_{2}\right)$ and $a_{3} \in D_{O U T}\left(m_{3}\right)$, then $m_{1} \stackrel{a_{1}, a_{3}}{\longrightarrow} m_{3}$.

Since $D_{A O}\left(m_{1}\right) \subseteq D_{A}\left(m_{1}\right)$, according to Definition [6] if $\left(a_{2}, a_{3}\right) \in D_{A O}\left(m_{1}\right)$, and $a_{3} \in D_{\text {OUT }}\left(m_{2}\right)$, then $m_{1} \stackrel{a_{1}, a_{2}}{\longrightarrow} m_{3}$. Thus, we have Corollary 1

Corollary 1. Let $m_{1}, m_{2}, m_{3}$ be modules and $a_{1}, a_{2}, a_{3}$ be attributes in an aspect. If $m_{1} \stackrel{a_{1}, a_{2}}{\longrightarrow} m_{2}$ and $m_{2} \stackrel{a_{2}, a_{3}}{\longrightarrow} m_{3}$, then $m_{1} \stackrel{a_{1}, a_{3}}{\longrightarrow} m_{3}$.

Property 4. Let $m_{1}, m_{2}, m_{3}$ be modules and $a_{1}, a_{2}$ be attributes in an aspect. If $m_{1} \stackrel{*, *}{\longrightarrow} m_{2}$ and $m_{2} \stackrel{a_{1}, a_{2}}{\longrightarrow} m_{3}$, then $m_{1} \stackrel{a_{1}, a_{2}}{\longrightarrow} m_{3}$.

From Properties 24, we can define the inter-module transitive dependence as follows.

Definition 7. Let $A$ be an aspect, $m_{i}(i>0)$ be modules, and $a_{i}(i>0)$ be attributes in $A$. If there exist modules $m_{1}, \ldots, m_{n}$ and attributes $a_{1}, \ldots, a_{n}(n>$ $1, a_{i}$ need not be unique, and $a_{i}$ may be " $*$ ", which models calls between modules), where $m_{1} \stackrel{a_{1}, a_{2}}{\longrightarrow} m_{2}, \ldots, m_{i-1} \stackrel{a_{i-1}, a_{i}}{\longrightarrow} m_{i}, \ldots, m_{n-1} \stackrel{a_{n-1}, a_{n}}{\longrightarrow} m_{n}$, then $m_{1}$ is intermodule-transitive-dependent on $m_{n}$, denoted by $m_{1} \stackrel{*}{\longrightarrow} m_{n}$.

To present our cohesion measure in a unified model, we introduce the aspect dependence graph to explicitly represent all types of dependencies in an aspect.

Definition 8. The aspect dependence graph (ADG) of an aspect $A$ is a directed graph $5, G_{A D G}=(V, A, T)$ where $V=V_{a} \cup V_{m}, A=A_{a a} \cup A_{m m} \cup A_{m a}$, and $T \in A \times\left(V^{\prime}, V^{\prime}\right)$ are the sets of vertex, arc, and tag respectively, such that:

- $V_{a}$ is the set of attribute vertices: each represents a unique attribute (the name of a vertex is the name of the attribute it represents) in $A$.

- $V_{m}$ is the set of module vertices: each represents a unique module (the name of a vertex is the name of the module it represents) in $A$.

- $V^{\prime}$ is the union of $V_{a}$ and $\{*\}$, i.e., $V^{\prime}=V_{a} \cup\{*\}$.

- $A_{a a}$ is the set of inter-attribute dependence arcs that represents dependencies between attributes, such that for $v_{a}, v_{a^{\prime}} \in V_{a}$ if $a \hookrightarrow a^{\prime}$, then $\left(v_{a}, v_{a^{\prime}}\right) \in A_{a a}$.

- $A_{m m}$ is the set of inter-module dependence arcs that represent dependencies between modules, such that for $v_{m}, v_{m^{\prime}} \in V_{m}$ if $m \rightarrow m^{\prime}$, then $\left(v_{m}, v_{m^{\prime}}\right) \in$ $A_{m m}$.

${ }^{5}$ A directed graph $G=(V, A)$, where $V$ is a set of vertices and $A \in V \times V$ is a set of arcs. Each $\operatorname{arc}\left(v, v^{\prime}\right) \in A$ is directed from $v$ to $v^{\prime}$; we say that $v$ is the source and $v^{\prime}$ the target of the arc. 
- $A_{m a}$ is the set of module-attribute dependence arcs that represents dependencies between modules and attributes, such that for $v_{m} \in V_{m}, v_{a} \in V_{a}$ if $m \mapsto a$, then $\left(v_{m}, v_{a}\right) \in A_{m a}$.

Generally, $G_{A D G}$ consists of three sub-graphs, i.e., inter-attribute dependence graph $G_{A A G}=\left(V_{a}, A_{a a}\right)$, module-attribute dependence graph $G_{M A G}=\left(V, A_{m a}\right)$, and inter-module dependence graph $G_{M M G}=\left(V_{m}, A_{m m}, T\right)$, which can be used to define the inter-attribute, module-attribute, and inter-module cohesion in an aspect respectively. Fig. 2 shows the $G_{M A G}$ and $G_{M M G}$ of aspect PS_Protocol. Since there exists no inter-attribute dependence in the aspect, the $G_{A A G}$ is not available. Note that we omit the Tags in both graphs for convenience.

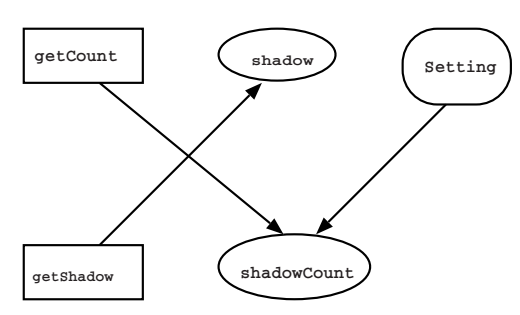

(a)

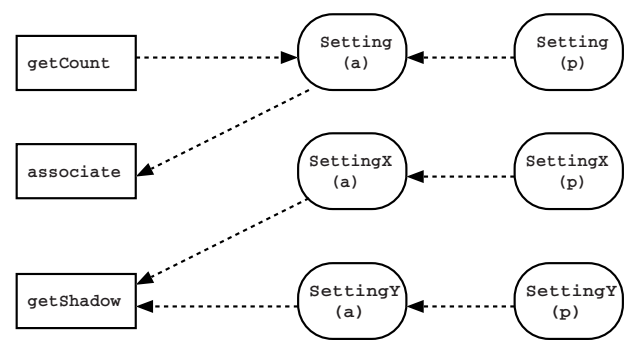

(b)

Fig. 2. The $G_{M A G}$ (a) and $G_{M M G}$ (b) of the aspect PS_Protocol in Fig. 1.

\section{Measuring Aspect Cohesion}

Briand et al. 4 have stated that a good cohesion measure should have properties such as non-negative and standardization, minimum and maximum, monotony, and cohesion does not increase when combining two components. We believe that these properties provide also a useful guideline even in aiding the development of an aspect cohesion measure. In this section, we propose our aspect cohesion measures, and show that our aspect cohesion measures satisfy the properties given by Briand et al. 4.

An aspect consists of attributes and modules such as advice, introduction, pointcuts, and methods. There are three types of dependencies between attributes and/or modules. Thus, the cohesion of an aspect should be measured from the three facets. In the following discussion, we assume that an aspect $A$ consists of $k$ attributes and $n$ modules, where $k, n \geq 0$.

\subsection{Measuring Inter-attribute Cohesion}

Inter-attribute cohesion is about the tightness between attributes in an aspect. To measure the inter-attribute cohesion for an aspect $A$, for each attribute $a$ of $A$, we introduce a set $D_{a}$ to record the attributes on which $a$ depends, i.e., $D_{a}(a)=\left\{a \mid a_{1} \stackrel{*}{\hookrightarrow} a, a_{1} \neq a\right\}$. Thus, we define the inter-attribute cohesion of $A$ as: 


$$
\gamma_{a}(A)= \begin{cases}0 & \mathrm{k}=0 \\ 1 & \mathrm{k}=1 \\ \frac{1}{k} \sum_{i=1}^{k} \frac{\left|D_{a}\left(a_{i}\right)\right|}{k-1} & k>1\end{cases}
$$

where $k$ is the number of attributes in $A$, and $\frac{\left|D_{a}\left(a_{i}\right)\right|}{k-1}$ represents the degree on which $a_{i}$ depends on other attributes in $A$.

If $k=0$, there is no attribute in $A$. Inter-attribute cohesion is useless, thus we set $\gamma_{a}(A)=0$. If $k=1$, there is only one attribute in $A$. Although it can not depend on other attribute, it itself is tight, thus we set $\gamma_{a}(A)=1$. If each attribute relates to all others, then $\gamma_{a}(A)=1$. If all attributes can exist independently, then $\gamma_{a}(A)=0$. Thus, $\gamma_{a}(A) \in[0,1]$.

Theorem 1. Let $A$ be an aspect and $G_{A A G}=\left(V_{a}, A_{a a}\right)$ be the inter-attribute dependence graph of $A . \gamma_{a}(A)$ does not decrease when adding an arc $\left(a_{1}, a_{2}\right) \in$ $A_{a a}$, where $a_{1}, a_{2} \in V_{a}$, on $G_{A A G}$.

Proof: Let $D_{a}\left(a_{1}\right)$ be the set of attributes in $A$ which $a_{1}$ depends on before adding an arc. We use $D_{a}^{\prime}\left(a_{1}\right)$ to represent $D_{a}\left(a_{1}\right)$ after adding an $\operatorname{arc}\left(a_{1}, a_{2}\right)$ to $D_{a}\left(a_{1}\right)$.

(1) If $a_{2} \in D_{a}\left(a_{1}\right)$, according to the definition of $D_{a}, a_{1}$ transitively depends on $a_{2}$, $D_{a}\left(a_{1}\right)$ does not change when adding arc $\left(a_{1}, a_{2}\right)$, i.e., $D_{a}\left(a_{1}\right)=D_{a}^{\prime}\left(a_{1}\right), \gamma_{a}(A)$ keeps unchanged;

(2) If $a_{2} \notin D_{a}\left(a_{1}\right)$, according to the definition of $D_{a}, D_{a}^{\prime}\left(a_{1}\right)$ will increase after adding arc $\left(a_{1}, a_{2}\right)$, i.e., $D_{a}^{\prime}\left(a_{1}\right)=D_{a}\left(a_{1}\right) \cup\left\{a_{2}\right\}$. For other attributes that depend on $a_{1}$, they will transitively depend on $a_{2}$, after adding $\operatorname{arc}\left(a_{1}, a_{2}\right)$. In all, $\gamma_{a}(A)$ will increase.

Therefore, $\gamma_{a}(A)$ does not decrease when adding an arc on $G_{A A G}$.

Theorem 2. Let $A_{1}$ and $A_{2}$ be two aspects and $A_{12}$ be an aspect derived from the combination of $A_{1}$ and $A_{2}$. Let $\gamma_{a}\left(A_{1}\right)$ and $\gamma_{a}\left(A_{2}\right)$ be the inter-attribute cohesions of $A_{1}$ and $A_{2}$ respectively and $\gamma_{a}\left(A_{12}\right)$ be the inter-attribute cohesion of $A_{12} \cdot \gamma_{a}\left(A_{12}\right) \leq \max \left\{\gamma_{a}\left(A_{1}\right), \gamma_{a}\left(A_{2}\right)\right\}$.

Proof: When combining the two aspects, the previous dependencies do not change in the new aspect, and there is no new dependence added. Let $A_{1}$ and $A_{2}$ be two aspects that have $k_{1}$ and $k_{2}$ attributes respectively.

(1) If $k_{1}=k_{2}=0$, then $\gamma_{a}\left(A_{12}\right)=\gamma_{a}\left(A_{1}\right)=\gamma_{a}\left(A_{2}\right)=0$.

(2) If $k_{1}=1$ or $k_{2}=1$, we assume $k_{1}=1$, then $\gamma_{a}\left(A_{1}\right)=1$. Because this is the maximum of the cohesion, $\gamma_{a}\left(A_{12}\right)$ is no greater than $\gamma_{a}\left(A_{1}\right)$.

(3) If $k_{1}, k_{2}>1, k_{1}+k_{2}>k_{1}$ and $k_{1}+k_{2}>k_{2}$. For each attribute $a_{1}$ of $A_{1}$, we have $\frac{\left|D_{a}\left(a_{1}\right)\right|}{k_{1}-1} \geq \frac{\left|D_{a}\left(a_{1}\right)\right|}{k_{1}+k_{2}-1}$. For each attribute $a_{2}$ of $A_{2}$, we have $\frac{\left|D_{a}\left(a_{2}\right)\right|}{k_{2}-1} \geq \frac{\left|D_{a}\left(a_{2}\right)\right|}{k_{1}+k_{2}-1}$. Thus, $\gamma_{a}\left(A_{12}\right) \leq \max \left\{\gamma_{a}\left(A_{1}\right), \gamma_{a}\left(A_{2}\right)\right\}$.

In all the cases above, inter-attribute cohesion does not increase when combining two aspects.

Example. The $D_{a}$ sets for each module in PS_Protocol are: $D_{a}$ (shadowCount) = $D_{a}($ shadow $)=\emptyset$. Therefore, $\gamma_{a}($ PS_Protocol $)=\frac{1}{2} \sum_{i=1}^{2} \frac{\left|D_{a}\left(a_{i}\right)\right|}{2-1}=0$. 


\subsection{Measuring Module-Attribute Cohesion}

Module-attribute cohesion is about the tightness between modules and attributes in an aspect. To measure this kind of cohesion, for each module $m$ in an aspect $A$, we introduce two sets: $D_{m a}$ and $D_{m a}^{o}$, where

- $D_{m a}(m)$ is the set of all $A$ 's attributes that are referred in $m$.

- $D_{m a}^{o}(m)$ is a set of all $A$ 's attributes that are referred in $m$ and related to attributes referred in other modules, i.e.,

$D_{m a}^{o}(m)=\left\{a \mid \exists a_{1}, m_{1}\right.$ such that $\left(\left(m_{1} \stackrel{a_{1}, a}{\longrightarrow} m\right) \vee\left(m \stackrel{a, a_{1}}{\longrightarrow} m_{1}\right)\right) \wedge\left(a, a_{1} \neq\right.$ $\left.\left.{ }^{\prime} *^{\prime}\right)\right\}$.

Obviously, $D_{m a}^{o}(m) \subseteq D_{m a}(m)$. We can define the module-attribute cohesion for $A$ as follows:

$$
\gamma_{m a}(A)= \begin{cases}0 & \mathrm{n}=0 \\ 1 & \mathrm{n}=1 \text { and }\left|D_{m a}\left(m_{i}\right)\right| \neq 0 \\ \frac{1}{n} \sum_{i=1}^{n} \frac{\left|D_{m a}^{o}\left(m_{i}\right)\right|}{\left|D_{m a}\left(m_{i}\right)\right|} & \text { others }\end{cases}
$$

where $n$ is the number of modules in $A$, and $\frac{\left|D_{m a}^{o}\left(m_{i}\right)\right|}{\left|D_{m a}\left(m_{i}\right)\right|}$, denoted by $\rho\left(m_{i}\right)$, is the ratio between the number of attributes which are referred in $m_{i}$ and relevant to others, to the number of all attributes referred in $m_{i}$.

For a module $m$, if $D_{m a}(m)=\emptyset$, i.e., no attribute is referred in $m$, we set $\rho(m)=0$. If the attributes referred in $m$ are not related to other modules, these attributes can work as local variables. It decreases the cohesion to take a local variable for a module as an attribute for all modules. If there is no attribute or module in the aspect, no module will depend on others. There is no $D_{m a}$ or all the $D_{m a}$ are empty, i.e., $\left|D_{m a}(m)\right|=0$. Thus, $\gamma_{m a}=0$. If each attribute referred in $m$ is related to other modules, then $\rho(m)=1$.

Theorem 3. Let $A$ be an aspect and $G_{M M G}=\left(V_{m}, A_{m m}\right)$ be the inter-module dependence graph of $A$. Let $m_{1}$ be a module of $A$ and $\rho\left(m_{1}\right)=\frac{\left|D_{m a}^{o}\left(m_{1}\right)\right|}{\left|D_{m a}\left(m_{1}\right)\right|} \cdot \rho\left(m_{1}\right)$ does not decrease when adding an arc $\left(m_{1}, m_{2}\right)$, where $m_{1}, m_{2} \in V_{m}$, on $G_{M M G}$.

Proof: Let the arc added have the form $m_{1} \stackrel{a_{1}, a_{2}}{\longrightarrow} m_{2}$, the attribute set $m_{1}$ refers is $D_{m a}\left(m_{1}\right)$ before adding the arc, among which the attributes related to other modules are included in the set $D_{m a}^{o}\left(m_{1}\right)$. They change to $D_{m a}^{\prime}\left(m_{1}\right)$ and $D_{m a}^{o}{ }^{\prime}\left(m_{1}\right)$ after adding the arc.

(1) If $a \in D_{m a}^{o}\left(m_{1}\right)$, according to the definitions, since the two sets do not change when adding an arc, $\rho\left(m_{1}\right)$ does not change.

(2) If $a \notin D_{m a}^{o}\left(m_{1}\right)$ and $a \in D_{m a}\left(m_{1}\right), D_{m a}\left(m_{1}\right)$ will not be changed when adding the arc, but $D_{m a}^{o}\left(m_{1}\right)$ will be increased, i.e. $D_{m a}^{o}{ }^{\prime}\left(m_{1}\right)=D_{m a}^{o}\left(m_{1}\right) \cup\{a\}$. Thus $\rho\left(m_{1}\right)$ will increase.

(3) If $a \notin D_{m a}\left(m_{1}\right)$, since $D_{m a}^{o}\left(m_{1}\right) \subseteq D_{m a}\left(m_{1}\right), a \notin D_{m a}^{o}\left(m_{1}\right)$. Therefore, the two sets will increase after adding the arc, i.e., $D_{m a}^{o}{ }^{\prime}\left(m_{1}\right)=D_{m a}^{o}\left(m_{1}\right) \cup\{a\}$, and $D_{m a}^{\prime}\left(m_{1}\right)=D_{m a}\left(m_{1}\right) \cup\{a\}$. Then $\left|D_{m a}^{o}{ }^{\prime}\left(m_{1}\right)\right|=\left|D_{m a}^{o}\left(m_{1}\right)\right|+1$, and $\left|D_{m a}^{\prime}\left(m_{1}\right)\right|=$ $\left|D_{m a}\left(m_{1}\right)\right|+1$. Since $\frac{\left|D_{m a}^{o}\left(m_{1}\right)\right|}{\left|D_{m a}\left(m_{1}\right)\right|} \leq \frac{\left|D_{m a}^{o}\left(m_{1}\right)\right|+1}{\left|D_{m a}\left(m_{1}\right)\right|+1}, \rho\left(m_{1}\right)$ does not decrease.

(4) If the added arc is an inter-module call dependence arc, we have $D_{m a}^{o}{ }^{\prime}\left(m_{1}\right)=$ $D_{m a}^{o}\left(m_{1}\right) \cup D_{m a}^{o}\left(m_{2}\right)$ and $D_{m a}^{\prime}\left(m_{1}\right)=D_{m a}\left(m_{1}\right) \cup D_{m a}\left(m_{2}\right)$. Applying these relations to cases (1) - (3), we will have the same conclusions. 
Similarly, we can prove the conclusion holds if the arc added is $m_{2} \stackrel{a_{1}, a_{2}}{\longrightarrow} m_{1}$.

Example. The $D_{m a}$ and $D_{m a}^{o}$ sets for each module in PS_Protocol are $D_{m a}$ (getShadow) $=\{$ shadow $\}, D_{m a}($ getCount $)=D_{m a}($ setting $)=\{$ shadowCount $\}, D_{m a}($ associate $)=$ $D_{m a}($ settingX $)=D_{m a}($ settingY $)=\emptyset, D_{m a}^{o}($ getCount $)=D_{m a}^{o}($ getShadow $)=\emptyset$, $D_{m a}^{o}($ associate $)=D_{m a}^{o}($ setting $)=\emptyset$, and $D_{m a}^{o}($ settingX $)=D_{m a}^{o}($ settingY $)=\emptyset$. Therefore, $\gamma_{m a}$ (PS_Protocol) $=\frac{1}{6} \sum_{i=1}^{6} \frac{\left|D_{m a}^{o}\left(m_{i}\right)\right|}{\left|D_{m a}\left(m_{i}\right)\right|}=0$.

\subsection{Measuring Inter-module Cohesion}

In the $G_{M M G}$, although the modules can be connected by attributes, this is not necessary sure that these modules are related. If there does exist some relations between modules, we should determine their tightness. This is the process to measure the inter-module cohesion. To do this, we introduce another set $D_{m}$ for each module $m$ in an aspect $A$, where $D_{m}(m)=\left\{m_{2} \mid m_{1} \stackrel{*}{\longrightarrow} m_{2}\right\}$.

The inter-module cohesion $\gamma_{m}(A)$ for $A$ is defined as follows:

$$
\gamma_{m}(A)= \begin{cases}0 & \mathrm{n}=0 \\ 1 & \mathrm{n}=1 \\ \frac{1}{n} \sum_{i=1}^{n} \frac{\left|D_{m}\left(m_{i}\right)\right|}{n-1} & n>1\end{cases}
$$

where $n$ is the number of modules in $A$ and $\frac{\left|D_{m}\left(m_{i}\right)\right|}{n-1}$ represents the tightness between $m_{i}$ and other modules in $A$. If each module depends on all other modules, then $\gamma_{m}(A)=1$. If all modules are independent, i.e., each module has no relation with any other modules, then $\gamma_{m}(A)=0$.

Theorem 4. Let $A$ be an aspect, $G_{M M G}=\left(V_{m}, A_{m m}\right)$ be the inter-module dependence graph of $A$. The inter-module cohesion $\gamma_{m}(A)$ does not decrease when adding an arc $\left(m_{1}, m_{2}\right) \in A_{m m}$, where $m_{1}, m_{2} \in V_{m}$, on $G_{M M G}$.

Theorem 5. Let $A_{1}$ and $A_{2}$ be aspects and $A_{12}$ be an aspect derived from the combination of $A_{1}$ and $A_{2}$. Let $\gamma_{m}\left(A_{1}\right)$ and $\gamma_{m}\left(A_{2}\right)$ be the inter-module cohesions of $A_{1}$ and $A_{2}$ and $\gamma_{m}\left(A_{12}\right)$ be the inter-module cohesion of $A_{12} \cdot \gamma_{m}\left(A_{12}\right) \leq$ $\max \left\{\gamma_{m}\left(A_{1}\right), \gamma_{m}\left(A_{2}\right)\right\}$.

We can prove Theorems 4 and [5 with a similar way as we did for Theorems 1 and 2. Due to the limitation of the space, we do not repeat them here.

Example. The $D_{m}$ sets for each module in PS_Protocol are: $D_{m}$ (getCount) = $\{$ setting $\}, D_{m}($ getShadow $)=\{$ shadow $\}, D_{m}($ setting $)=\{$ associate $\}, D_{m}($ settingX $)$ $=\{$ getShadow $\}$, and $D_{m}$ (settingY $)=\{$ getShadow $\}$. Therefore, $\gamma_{m}($ PS_Protocol $)=$ $\frac{1}{6} \sum_{i=1}^{6} \frac{\left|D_{m}\left(m_{i}\right)\right|}{|6-1|}=\frac{1}{6}$.

\subsection{Measuring Aspect Cohesion}

After measuring the three facets of aspect cohesion independently, we have a discrete view of the cohesion of an aspect. We have two ways to measure the aspect cohesion for an aspect $A$ : 
(1) Each measurement works as a field. The aspect cohesion for $A$ is a 3-tuple as $\Gamma(A)=\left(\gamma_{a}, \gamma_{m a}, \gamma_{m}\right)$.

(2) Integrating the three facets as a whole. Let $x=\beta_{1} * \gamma_{a}+\beta_{2} * \gamma_{m a}+\beta_{3} * \gamma_{m}$, the aspect cohesion for $A$ is computed as follows.

$$
\Gamma(A)=\left\{\begin{array}{ll}
0 & \mathrm{n}=0 \\
\beta * \gamma_{m} & \mathrm{k}=0 \\
x & \text { others }
\end{array} \text { and } n \neq 0\right.
$$

where $k$ is the number of attributes and $n$ is the number of modules in $A$, $\beta \in(0,1], \beta_{1}, \beta_{2}, \beta_{3}>0$, and $\beta_{1}+\beta_{2}+\beta_{3}=1$.

If $k=0$ and $n \neq 0, \Gamma(A)$ describes only the tightness of the call relations, thus we introduce a parameter $\beta$ to constrain it. For other cases, we introduce three parameters $\beta_{1}, \beta_{2}$, and $\beta_{3}$ to constrain it. The selection of $\beta_{1}, \beta_{2}$, and $\beta_{3}$ is determined by users.

Example. The aspect cohesion of PS_Protocol can be computed based on its $\gamma_{a}, \gamma_{m a}$, and $\gamma_{m}$. If we set $\beta_{1}=\beta_{2}=\beta_{3}=\frac{1}{3}$, we have $\Gamma$ (PS_Protocol $)=\frac{1}{3} * \gamma_{a}$ (PS_Protocol) + $\frac{1}{3} * \gamma_{m a}$ (PS_Protocol) $+\frac{1}{3} * \gamma_{m}$ (PS_Protocol $)=\frac{1}{18}$.

\section{Related Work}

We discuss some related work that directly or indirectly influences our work presented in this paper. To the best of our knowledge, our work is the first attempt to study how to assess the cohesion of aspects in aspect-oriented software.

The approaches taken to measure cohesiveness of procedural programs have generally tried to evaluate cohesion on a procedure (function) by procedure (function) basis. Bieman and Ott [3] propose an approach to measuring the cohesion on procedures based on a relation between output tokens (output variables) and program slices. Kang and Bieman 11 investigate to measure cohesion at the design level for the case that the code has yet to be implemented. Since aspects are more complex and significantly different abstractions in comparing with procedures (functions), These measures definitely fails to be applied to aspects.

Most existing approaches for class cohesion measurement consider the interactions between methods and/or attributes in a class. Chidamber and Kemerer 8] propose the Lack of Cohesion Measure (LCOM) to assess class cohesion based on the similarity of two methods in a class. Hitz and Montazeri [10] propose an extension to the LCOM of Chidamber and Kemerer by making it more sensitive to small changes in the structure of a class. Chae, Kwon, and Bae [6] propose a class cohesion measure for object-oriented system by introducing a new notion called glue methods. In contrast to the above cohesion measurement approaches that only consider the interaction between methods and/or attributes, Chen et al. [7] propose an approach to measuring class cohesion based on the interactions between attributes and/or methods in a class. Although their work is similar to ours, we see our work differing from theirs because our approach can handle interactions between attributes and those modules such as aspect advice, introduction, and pointcuts that are unique constructs for aspect-oriented programs. 
Based on these new types of interactions we propose a new dependence model for aspects that is different from the dependence model presented by Chen et al. [7].

Zhao [16] proposes a metrics suite for aspect-oriented software, which are specifically designed to quantify the information flows in aspect-oriented programs. To this end, Zhao presents a dependence model for aspect-oriented software which is composed of several dependence graphs to explicitly represent dependence relationships in a module, a class, or the whole program. Based on the model, Zhao defines some metrics to measure the complexity of an aspectoriented program from various different viewpoints and levels. However, Zhao does not address the issue of aspect cohesion measurement.

The development of coupling measures for aspect-oriented software is also considered by Zhao who proposes an approach to assessing the coupling of aspect-oriented software based on the interactions between aspects and classes 18.

Dufour et al. 9] proposes some dynamic metrics for AspectJ, which focuses on the performance and execution time costs, rather than structural complexity of aspect-oriented software.

\section{Concluding Remarks}

In this paper, we proposed an approach to measuring the cohesion of aspects in aspect-oriented software based on dependence analysis. We discussed the tightness of an aspect from three facets: inter-attribute, module-attribute and intermodule. These three facets can be used to measure the aspect cohesion independently and also can be integrated as a whole. We also discussed the properties of these dependencies and according to these properties we proved that our cohesion measures satisfy some properties which a good measure should have. Therefore, we believe our approach may provide a solid foundation for measuring aspect cohesion.

The aspect cohesion measures proposed in this paper focused only on the features of an aspect itself, and did not take its application environment into account. When do so, there will be a little difference because the modules in the aspect may be invoked in a set of given sequences, which is a subset of the arbitrary combination. For such a case, we should analyze the definitions and uses of attributes in the context of the applications. Also, in this paper we did not distinguish the connected and non-connected graphs and did not consider to measure the cohesion of a derived aspect (i.e., aspect inheritance). In our future work, we will study the influence of aspect inheritance and other aspect-oriented features on aspect cohesion, and apply our cohesion measure approach to real aspect-oriented software design.

\section{Acknowledgments}

This work was partially supported by the Japan Society for Promotion of Science (JSPS) under Grand-in-Aid for Scientific Research (C) (No.15500027). The authors would like to thank the anonymous referees for valuable comments on an earlier version of the paper. 


\section{References}

1. The AspectJ Team. The AspectJ Programming Guide. 2002.

2. L. Bergmans and M. Aksits. Composing crosscutting Concerns Using Composition Filters. Communications of the ACM, Vol.44, No.10, pp.51-57, October 2001.

3. J. Bieman and L. Ott. Measuring Functional Cohesion. IEEE Transactions on Software Engineering, Vol.22, No.10, pp.644-657, August 1994.

4. L.C. Briand, J. Daly and J. Wuest. A Unified Framework for Cohesion Measurement in Object-Oriented Systems. Empirical Software Engineering, Vol.3, No.1, pp.65-117, 1998.

5. L.C. Briand, S. Morasca and V.R. Basili. Defining and Validating Measures for Object-Based High-Level Design. IEEE Transactions on Software Engineering, Vol.25, No.5, pp.724-743, 1999.

6. H.S. Chae, Y. R. Kwon and D. H. Bae. A Cohesion Measure for Object-Oriented Classes. Software Practice \& Experience, Vol.30, No.12, pp.1405-1431, 2000.

7. Z. Chen, Y. Zhou, B. Xu, J. Zhao, and H. Yang. A Novel Approach to Measuring Class Cohesion Based on Dependence Analysis. Proceedings of the International Conference on Software Maintenance, October 2002.

8. S. R. Chidamber and C. F. Kemerer. A Metrics Suite for Object-Oriented Design. IEEE Transactions on Software Engineering, Vol.20, No.6, pp.476-493, 1994.

9. B. Dufour, C. Goard, L. Hendren, C. Verbrugge, O. de Moor, and G. Sittampalam. Measuring the Dynamic Behavior of AspectJ Programs. Scable Technical Report No.2003-8, McGill University and Oxford University, December 2003.

10. M. Hitz and B. Montazeri. Measuring Coupling and Cohesion in Object-Oriented Systems. Proceedings of International Symposium on Applied Corporate Computing, pp.25-27, Monterrey, Mexico, October 1995.

11. B. Kang and J. Bieman. Design-Level Cohesion Measures: Derivation, Comparison, and Applications. Computer Science Technical Report CS-96-103, Colarado State University, 1996.

12. G. Kiczales, J. Lamping, A. Mendhekar, C. Maeda, C. Lopes, J. M. Loingtier, and J. Irwin. Aspect-Oriented Programming. Proceedings of the 11th European Conference on Object-Oriented Programming, pp.220-242, LNCS, Vol.1241, SpringerVerlag, June 1997.

13. K. Lieberher, D. Orleans, and J. Ovlinger. Aspect-Oriented Programming with Adaptive Methods. Communications of the ACM, Vol.44, No.10, pp.39-41, October 2001.

14. P. Tarr, H. Ossher, W. Harrison, and S. M. Sutton. N Degrees of Separation: MultiDimensional Separation of Concerns. Proceedings of the International Conference on Software Engineering, pp.107-119, 1999.

15. E. Yourdon and L. Constantine. Structural Desing. Englewood Cliffs, Prentice Hall, 1979.

16. J. Zhao. Toward A Metrics Suite for Aspect-Oriented Software. Technical Report SE-136-5, Information Processing Society of Japan (IPSJ), March 2002.

17. J. Zhao and M. Rinard. System Dependence Graph Construction for AspectOriented Programs. Technical Report MIT-LCS-TR-891, Laboratory for Computer Science, MIT, March 2003.

18. J. Zhao. Coupling Measurement in Aspect-Oriented Systems. Technical Report SE-142-6, Information Processing Society of Japan (IPSJ), June 2003. 